\title{
Cenani-Lenz syndrome
}

INSERM

\section{Source}

INSERM. (1999). Orphanet: an online rare disease and orphan drug data base. CenaniLenz syndrome. ORPHA:3258

Cenani-Lenz syndrome (CLS) is a congenital malformation syndrome that associates a complex syndactyly of the hands with malformations of the forearm bones and similar manifestations in the lower limbs. 